This article was published in Journal of Power Sources, 273, 423-430, 2015

http://dx.doi.org/10.1016/j.jpowsour.2014.09.093

\title{
Thermodynamic Analysis of Glycerol Steam Reforming for Hydrogen Production with in Situ Hydrogen and Carbon Dioxide Separation
}

\author{
Joel M. Silva ${ }^{l}$, M. A. Soria ${ }^{1, *}$, Luis M. Madeira ${ }^{l}$
}

${ }^{1}$ LEPABE, Department of Chemical Engineering, Faculty of Engineering, University of Porto, Rua Dr. Roberto Frias s/n, 4200-465 Porto, Portugal

* Corresponding Author. Phone/Email: +351-225081519 / masoria@,fe.up.pt (M. A. Soria). 


\section{Abstract}

A thermodynamic study of Glycerol Steam Reforming (GSR) for hydrogen production with in situ carbon dioxide and hydrogen (reaction products) simultaneous removal was performed. The sorption-enhanced membrane reactor (SEMR) was divided into multiple subGibbs reactors and the Gibbs free energy minimization method was employed. The effects of temperature (600-800 K), molar water-to-glycerol feed ratio (WGFR) (3-9), pressure (1-5 atm) and fraction of hydrogen and carbon dioxide removal $(f, 0-0.99)$ on the GSR process were target of investigation. A hydrogen yield (total moles of hydrogen produced/mole of reacted glycerol) very close to the stoichiometric value of 7 was obtained at $700 \mathrm{~K}$, WGFR of 9,1 atm and for $f_{\mathrm{CO}_{2}}=0.99$ and $f_{\mathrm{H}_{2}}=0.80$. This corresponds to an enhancement of $217 \%, 47 \%$ and $22 \%$ in terms of hydrogen yield comparatively to the traditional reactor (TR), sorption-enhanced reactor (SER) with carbon dioxide capture $\left(f_{\mathrm{CO}_{2}}=0.99\right)$ and membrane reactor $(\mathrm{MR})$ with hydrogen separation $\left(f_{H_{2}}=0.80\right)$, respectively. In terms of coke, its formation was only observed under WGFRs below the stoichiometric value of 3 .

Keywords: glycerol; steam reforming; thermodynamics; sorption-enhanced reactor; membrane reactor; sorption-enhanced membrane reactor. 


\section{Notation and Glossary}

\section{List of acronyms}

$\begin{array}{ll}\text { GSR } & \text { Glycerol Steam Reforming } \\ \text { MR } & \text { Membrane reactor } \\ \text { RWGS } & \text { Reverse water-gas-shift } \\ \text { SER } & \text { Sorption-Enhanced Reactor } \\ \text { SEMR } & \text { Sorption-Enhanced Membrane Reactor } \\ \text { SRM } & \text { Steam Reforming of Methane } \\ \text { TR } & \text { Traditional Reactor } \\ \text { WGFR } & \text { Water-to-Glycerol Feed Ratio } \\ \text { WGS } & \text { Water-Gas Shift }\end{array}$




\section{Introduction}

The use of glycerol, the main by-product of the biodiesel manufacture process, for hydrogen production is not only environmentally more attractive than fossil-based routes, but also valorizes glycerol itself thus making biodiesel more competitive. The Glycerol Steam Reforming (GSR) is an endothermic and equilibrium-limited process that requires high operating temperatures (eq. (1) - cf. Table 1). The GSR process involves the decomposition of glycerol (eq. (2) - cf. Table 1) followed by the water-gas shift (WGS, eq. (3) - cf. Table 1) reaction (multiplied by a factor of 3 ).

Even though a theoretical production of 7 moles of hydrogen per mole of consumed glycerol should be observed, the existence of side reactions (e.g. methanation) leads to a decrease in hydrogen production. Theoretical studies on new reactor configurations that combine GSR and carbon dioxide [1,2] or hydrogen [3] selective removal (reaction products in eq. (1)) have allowed to conclude that such intensified processes permit to enhance the hydrogen production and decrease the production of both methane and carbon monoxide by-products by shifting the thermodynamic equilibrium. Moreover, such processes allow the attainment of similar or even better performance than in a traditional reactor (TR) while operating at milder operating conditions. However, the effect of the selective removal of both products simultaneously during GSR has never been studied. Such a process could be achieved with a multifunctional Sorption Enhanced Membrane Reactor (SEMR), which would simultaneously carry out the GSR reaction and remove pure hydrogen and carbon dioxide from the reaction zone by means of a hydrogen selective membrane and a carbon dioxide selective sorbent, respectively (Fig. 1). The continuous use of such reactor configuration would require two parallel reactors, being that while one of them is producing pure $\mathrm{H}_{2}$ through GSR (which exits in the permeate stream and is not mixed with any other species, apart from the inert carrier gas, due to membrane selective permeation - left side of Fig. 1), the other is being regenerated, i.e., carbon dioxide is being removed and exits in the retentate stream (again $\mathrm{N}_{2}$ can be used as purge gas - right side of Fig. 1). In other words, each 
reactor has two operation modes being that along time each of them goes through the reactionregeneration cycle repeatedly. Since the goal is to shift the thermodynamic equilibrium of GSR during reaction mode by retaining $\mathrm{CO}_{2}$ in the sorbent, once the sorbent gets saturated and $\mathrm{CO}_{2}$ starts breaking through the column the reaction is ended (Fig. 1 (a)). At this point, the set of valves moves this reactor feed (glycerol and steam) to e.g. $\mathrm{N}_{2}$ so that the sorbent bed can be regenerated (Fig. 1 (b)). During this last step no $\mathrm{H}_{2}$ is produced in this reactor, being produced in the other reactor that is now in the reaction stage. Moreover, although $\mathrm{CO}_{2}$ might affect membrane permeability towards $\mathrm{H}_{2}$, this is not very pronounced as, for instance, it occurs with $\mathrm{CO}$, and such effect is reversible (thus, as long as $\mathrm{CO}_{2}$ is removed, high permeability towards $\mathrm{H}_{2}$ is restored) [4]. The main requirement is that operating conditions, namely in terms of temperature, are compatible for the GSR catalyst, $\mathrm{CO}_{2}$ sorbent and $\mathrm{H}_{2}$-selective membrane, which is the case [5].

In this work an equilibrium thermodynamic analysis of GSR in a SEMR was done, for the first time, with ASPEN PLUS software and the effect of the operating temperature, pressure, water-to-glycerol feed ratio (WGFR) and $\mathrm{H}_{2}$ removal fraction (for constant $\mathrm{CO}_{2}$ removal) on hydrogen production was accessed. Moreover, a comparison between the results obtained under equilibrium conditions for the SEMR and for the other types of reactors (TR, membrane reactor (MR) and sorption enhanced reactor (SER)) was done as well. Finally, the limits in terms of WGFR for avoiding coke formation at different temperatures was discussed. This thermodynamic analysis is crucial as it may indicate the suitability of applying such intensified reaction-separation process for hydrogen production from glycerol. Moreover, and although thermodynamic considerations alone do not provide evidence for the practicability of the design, it provides valuable information about the limits that can be attained in actual operation, and gives indication on how to proceed and which conditions have to be applied in order to achieve maximum performance. 


\section{Methodology}

ASPEN PLUS V7.3 was used to perform all the simulations. The Gibbs free energy minimization methodology (nonstoichiometric method), which calculates the equilibrium composition through the minimization of the Gibbs free energy of a specific set of species was applied (a more detailed description of the methodology is available in the supplementary data).

For such simulations, the species included were hydrogen, carbon dioxide, carbon monoxide, methane, glycerol, water and solid carbon. By considering these species, some of the possible reactions that might be in equilibrium together with GSR (eqs. (2 and 3)), are the ones shown in Table 1. The reaction in eq. (5), which is the sum of both reverse of eq. (3) and eq. (4), is included in Table 1 with the single purpose of facilitating the analysis of the stoichiometric relations in the SEMR.

The modular approach used to simulate the SEMR (supplementary data, Fig. S1) is represented by $(n+1)$ sub-reformers and $n$ sub-separators. Each sub-reformer consists on a Gibbs reactor which performs the thermodynamic equilibrium calculations through the method previously explained. The sub-separators consist on component separators that separate chemical species based on specified flows or split fractions, which in practice is related with factors like membrane and sorbent selectivities, membrane area and sorbent quantity, etc. For $n=0$ a simple Gibbs reactor is simulated; for $n=1$ a single separator and two sub-reformers are used; and so on. The value of $n$ depends on the values of the global removal fractions of $\mathrm{CO}_{2}\left(f_{\mathrm{CO}_{2}}\right)$ and $\mathrm{H}_{2}$ $\left(f_{\mathrm{H}_{2}}\right)$ specified (higher values of $f$ imply higher values of $n$ ). It is worth mentioning that a similar methodology has been used in previous works $[1,3]$.

The thermodynamic analysis was performed for temperatures in the range of $600-800 \mathrm{~K}$, pressures between 1-5 atm and WGFRs between 3-9 (realistic operation conditions for hydrogen selective membranes $[6,7]$ and carbon dioxide sorbents $[5,8,9]$, which are close to those typically employed in the GSR $[10,11])$; the study considered also hydrogen removal fractions in the range of 0-0.99 and a carbon dioxide removal fraction of 0.99 (for the reasons explained in 
a posterior section). For all the cases, the yields of hydrogen, methane, carbon dioxide and carbon monoxide were analyzed. These were defined as follows:

$$
Y_{i}=\left(n_{i, \text { separated }}+n_{i, \text { unseparated }}\right) / n_{\text {converted glycerol }}
$$

where $Y_{i}$ is the yield of species $i$ and $n_{\text {converted glycerol }}$ is the molar flow rate of glycerol that is converted into all reaction products.

\section{Results and Discussion}

\subsection{GSR with in situ hydrogen or carbon dioxide removal - comparison}

Before performing the thermodynamic equilibrium calculations for the SEMR, the thermodynamics of GSR performed in a TR, SER or MR was analyzed. In order to fully understand the effect of the simultaneous removal of both hydrogen and carbon dioxide during GSR on the thermodynamic equilibrium, it is essential to analyze first the effect of the individual removal of either hydrogen or carbon dioxide on the GSR thermodynamics. A comparison between both individual effects (hydrogen and carbon dioxide removal), which to the best of our knowledge has never been done before, is included as well.

Complete conversion of glycerol was observed for all the range of temperatures analyzed (data not shown), in agreement to what was observed by other authors [1-3, 12, 13]. This means that in the subsequent analysis eq. (2) is almost not taken into account as it is completely shifted towards reaction products. Coke formation was not observed for all the conditions used in this section, so eqs. (7-10) will now be discarded.

\subsubsection{Traditional Reactor $\left(f_{i}=0\right)$}

The variation with temperature of the dimensionless (normalized with the molar flow rate of glycerol fed to the reactor) molar flow rate of glycerol, water, hydrogen, carbon dioxide, carbon monoxide and methane in the thermodynamic equilibrium of a TR is presented in Fig. 2. 
In terms of hydrogen and carbon dioxide dimensionless molar flow rates, it can be seen that they increase with temperature up to a certain point, stabilize and then suffer a slight decrease (Fig. 2). In order to understand this behavior, the reactions in eqs. (3 and 4) should be considered. At lower temperatures ( $\sim 600 \mathrm{~K})$, the strongly exothermic methanation reaction (eq. (4)) is favored, in agreement with the maximum methane and water and minimum carbon monoxide dimensionless molar flow rates observed. Particularly, the dimensionless molar flow rate of water for such temperature is slightly higher than the value of 9 in the feed, thus indicating water formation. When temperature increases the methanation reaction is more disfavored (progressive inhibition of methane production as compared to WGS reaction (less exothermic - eq. (3)), which progressively becomes dominant $\left(\mathrm{CO}_{2}\right.$ and $\mathrm{H}_{2}$ production and $\mathrm{H}_{2} \mathrm{O}$ consumption). The stabilization of the hydrogen and carbon dioxide production at higher temperatures is related to the highly enhanced reverse WGS (RWGS), which leads to an increase in the water production. The faster stabilization of the carbon dioxide production observed (maximum at lower temperature than hydrogen) is due to the fact that while hydrogen is being consumed through RWGS and saved through the inhibition of methanation $\left(3\right.$ moles of $\mathrm{H}_{2}$ per mole of $\mathrm{CH}_{4}$ unproduced), carbon dioxide is only being consumed through RWGS. When the temperature is high enough so that there is no methane formation, the yield of hydrogen starts decreasing and the RWGS becomes the only dominant reaction (hydrogen and carbon dioxide decrease at a similar rate). Since carbon monoxide is consumed through WGS and methanation, which are both exothermic reactions, higher temperatures lead to higher carbon monoxide production. For the conditions here analyzed the material balances performed for each species considering only stoichiometry of reactions 2,3 and 4 were all consistent with the simulations results, thus indicating that there are no other reactions occurring besides the ones previously indicated.

Regarding the dry reforming of methane (eq. (6)), suggested by Adhikari et. al [12] to be responsible for the behavior of carbon dioxide production, once it depends on both WGS and methanation (eqs. (3 and 4)), which have been target of discussion already, it is implicitly already analyzed here. 


\subsubsection{Sorption-enhanced reactor versus membrane reactor}

The variation of the yield of hydrogen, methane, carbon monoxide and carbon dioxide with temperature and carbon dioxide removal fraction (in the SER) and hydrogen removal fraction (in the MR) is presented in Fig. 3 and Fig. 4, respectively. Besides increasing with temperature, the yield of hydrogen increases with the fraction of carbon dioxide or hydrogen removal as well. It can be observed by comparing Fig. 3 (a) and Fig. 4 (a) that higher hydrogen production can be attained by selectively removing hydrogen from the reaction medium instead of carbon dioxide, especially at lower temperatures. This difference is associated to the inhibition of the formation of the main GSR by-product, methane. When carbon dioxide is removed from the reactor, the equilibrium of the WGS reaction (eq. (3)) is shifted towards the production of more carbon dioxide, and inherently hydrogen, and carbon monoxide consumption. Since carbon dioxide does not participate in the reversible methanation reaction (eq. (4)), its removal does not directly affect this reaction. Instead, it is only affected by the enhanced production of hydrogen and carbon monoxide consumption (opposite effects). On the other hand, when hydrogen is removed from the reactor, it does not only directly affect the WGS reaction but also the methanation reaction, thus inhibiting the last reaction to a higher extent, which converts hydrogen into methane. Therefore, higher hydrogen yields are obtained in the MR than in the SER at the expense of higher methane production inhibition (Fig. 3 (b) and Fig. 4 (b)).

As already stated, when there is no methane generation, higher hydrogen yields are reached, being that the temperature of maximum hydrogen yield depends on how fast methane production is completely inhibited. In the SER, this maximum occurs at approximately $950 \mathrm{~K}$ for all carbon dioxide removal fractions since carbon dioxide does not directly interfere in the methanation reaction. On the other hand, in the MR the temperature at which the maximum hydrogen yield is obtained decreases for increasing hydrogen removal fractions (e.g. $900 \mathrm{~K}$ for $40 \%$ hydrogen removal vs. $850 \mathrm{~K}$ for $80 \%$ hydrogen removal). This emphasizes once again the effect that hydrogen removal has on avoiding more intensively methane formation and thus, hydrogen consumption. 
A similar behavior as recorded for hydrogen is observed for the carbon dioxide yield in both reactors (Figs. 3c and 4c). However, on the contrary to hydrogen, the yield of carbon dioxide starts declining at a temperature bellow the one that completely inhibits methane production, for the reason already mentioned in the previous section. When methane production is completely inhibited, carbon dioxide, in the same way as hydrogen, is consumed through RWGS. Once again, in a SER this maximum happens approximately at the same temperature for all carbon dioxide removal fractions while in the MR the maximum's temperature decreases with increasing hydrogen removal fractions, for the same reasons presented for hydrogen. Regarding carbon monoxide, a similar behavior is observed in both SER and MR. Since carbon monoxide is consumed through WGS and methanation, which are both exothermic reactions, higher temperatures shift the opposite reactions, leading to higher carbon monoxide yields. Finally, when higher carbon dioxide or hydrogen removals are applied, more carbon monoxide is consumed through WGS and so lower carbon monoxide yields are obtained, as intended.

\subsection{GSR with in situ hydrogen and carbon dioxide simultaneous \\ removal}

In this section the effect of the simultaneous removal of hydrogen and carbon dioxide during GSR, as well as the influence of temperature, WGFR and pressure on the yield of hydrogen, methane, carbon monoxide and carbon dioxide is accessed. Moreover, an analysis of the boundaries of operating conditions for solid carbon formation is included as well. During the simulations for the SEMR, the carbon dioxide removal fraction was kept at 0.99 (approximate removal normally obtained before the breakthrough in a packed bed) while the hydrogen removal was varied between 0 (SER only) and 0.99 . Once again, complete glycerol conversion and no coke formation were observed for all the range of conditions analysed.

\subsubsection{Effect of Temperature}

The variation of the yield of hydrogen and methane with temperature and hydrogen removal fraction is presented in Fig. 5. Since the variation of the carbon dioxide yield with temperature 
follows a trend very similar to that of hydrogen, its graphic is not presented here for brevity reasons. The carbon monoxide yield is not included here as well since only residual amounts were observed. Constant WGFR and pressure of 9 and $1 \mathrm{~atm}$, respectively, were used since such conditions have been reported in many works [13-16] as the most favourable conditions for GSR. However, their effects will be analyzed separately in a later section.

As can be seen in Fig. 5, higher temperatures highly enhance the hydrogen yield and decrease the methane formation because of the exothermic nature of the main secondary reaction, methanation (eq. (4)), which takes place mainly at lower temperatures. Regarding the hydrogen removal fraction, it clearly enhances hydrogen production, especially at lower temperatures. For hydrogen and carbon dioxide removals of $99 \%$, the theoretical hydrogen yield of 7 is obtained for all the range of temperatures tested. However, such hydrogen removal in a membrane reactor operating at $1 \mathrm{~atm}$ is pratically impossible. Therefore, a hydrogen removal of $80 \%$ [17] is considered to be much more realistic while operating at $1 \mathrm{~atm}$. Under such conditions a hydrogen production of 5 and 7 moles/per mole of glycerol converted at $600 \mathrm{~K}$ and $800 \mathrm{~K}$, respectively, was obtained. Compared to the other reactor configurations (SER and MR), the SEMR allows higher hydrogen generation, especially at lower temperatures. Therefore, from the thermodynamic point of view, the use of such reactor configuration would be much more benefitial if temperatures around $600 \mathrm{~K}$ are used. However, this decreases reaction kinetics and hydrogen permeation, so that longer reactors and thus, longer and/or more permeable membranes, would be required.

In terms of methane formation, the maximum methane production of 0.5 moles $/$ mole of glycerol consumed for $f_{\mathrm{CO}_{2}}=0.99$ and $f_{\mathrm{H}_{2}}=0.80$ was observed at $600 \mathrm{~K}$ (no considerable methane at $750 \mathrm{~K})$ - Fig. 5, while for the SER with $f_{\mathrm{CO}_{2}}=0.99$ and for the MR with $f_{\mathrm{H}_{2}}=0.80$, maximum methane productions of 1.29 (Fig. 3b) and 1.09 (Fig. 4b) moles/mole of glycerol converted were obtained, respectively, for the same temperature. Regarding carbon dioxide, its yield varies with temperature and hydrogen removal fraction in a similar way as hydrogen (data not shown) since it is a product of the same reaction that origins hydrogen (WGS (eq. (3))). 
Moreover, since the base case here already considers a carbon dioxide removal fraction as high as 0.99 , which already leads to a very similar behavior between hydrogen and carbon dioxide yields in the SER (the effect of carbon dioxide removal outweights the effect of temperature Fig. 3), then it is expected that by adding hydrogen removal, the similarities between the behavior of both species yields increase. Finally, it is again worth stressing that only residual amounts of carbon monoxide $(<0.01 \mathrm{~mol} / \mathrm{mol}$ of converted glycerol) were observed during the simulations for all the range of temperatures and hydrogen removal fractions.

It was observed, for all the range of temperatures and hydrogen removal fractions under which methane formation occurs that the variation of temperature or hydrogen removal fraction resulted in variations of the moles of hydrogen 4 times higher than the variations of the moles of methane and carbon dioxide (absolute value). This clearly indicates that both WGS (eq. (3)) and SRM (reverse of eq. (4)) controll the GSR process in the SEMR. On the other hand, under conditions that do not allow methane formation (high hydrogen removal fractions and high temperatures), the process is solely controlled by the WGS reaction.

By comparing the SEMR with the other three reactor configurations in terms of hydrogen yield, it is clear that the SEMR presents better performance than both the SER and MR and much better performance than the TR. In fact, while in the TR, at $700 \mathrm{~K}, 1$ atm and WGFR of 9, a hydrogen yield of 2.18 was attained, in the SER with $f_{\mathrm{CO}_{2}}=0.99$, MR with $f_{\mathrm{H}_{2}}=0.80$ and SEMR with $f_{\mathrm{CO}_{2}}=0.99$ and $f_{\mathrm{H}_{2}}=0.80$, hydrogen yields of $4.69,5.66$ and 6.92 were observed, respectively, under the same conditions. This corresponds to an enhancement of the hydrogen yield of aproximately $115 \%$ when the SER with $f_{\mathrm{CO}_{2}}=0.99$ is used instead of the TR, an enhancement of approximately $21 \%$ when the MR with $f_{\mathrm{H}_{2}}=0.80$ is used instead of the SER with $f_{\mathrm{CO}_{2}}=0.99$ (enhancement of approximately $160 \%$ comparatively to the TR) and an increase of around $22 \%$ when the SEMR with $f_{\mathrm{CO}_{2}}=0.99$ and $f_{\mathrm{H}_{2}}=0.80$ is used instead of the MR with $f_{\mathrm{H}_{2}}=0.80$ (enhancement of $217 \%$ and $47 \%$ comparatively to the TR and the SER with $f_{\mathrm{CO}_{2}}=$ 0.99, respectively). 


\subsubsection{Effect of Water-to-Glycerol Feed Ratio}

The effect of both hydrogen removal fraction and WGFR on the yield of hydrogen and methane was target of investigation as well. Such study is depicted in Fig. 6. The WGFR was varied between 3 (stoichiometric value) and 9 , which has been considered by many authors as the most favorable value, as already mentioned. Higher values were not considered because this would imply, in practice, excessive costs for water vaporization.

In terms of hydrogen production, the increase of the WGFR enhances the production of hydrogen, especially at lower hydrogen removal fractions. By increasing the water content in the reaction environment, the thermodynamic equilibrium of both GSR and SRM (reverse of eq. (4)) is deslocated, according to the Le Chatelier's principle, towards the conversion of the excess of water into hydrogen. Thus, the methane yield decreases, as can be seen in Fig. 6b. Regarding the carbon dioxide yield, its behavior is very similar to hydrogen (data not shown) because of the reasons already stated in the previous section. Once again, only residual amounts of carbon monoxide were observed. The process is controlled by the reactions in eqs. $(2,3$ and 4$)$ for all the WGFRs involved. When there is no methane, only eqs. (2 and 3$)$ are relevant.

Concerning the effect of increasing hydrogen removal fractions, it is clear that higher removal fractions of hydrogen lead to higher hydrogen production and methane production inhibition. Moreover, this enhancement is more pronounced for less favorable conditions (WGFR $=3)$.

In sum, considering again the case in which $f_{\mathrm{CO}_{2}}=0.99$ and $f_{\mathrm{H}_{2}}=0.80$, when the WGFR increased from 3 to 9 , the hydrogen yield increased from 4.57 to $6.92 \mathrm{moles} / \mathrm{mole}$ of converted glycerol, while the yield of methane decreased from 0.61 to 0.02 .

If higher WGFRs had been tested, higher hydrogen yields would have been obtained, especially at lower hydrogen removal fractions. However, such water contents are considered to be disadvantageous due to the high vaporization costs involved at industrial scale. Moreover, even if they allow avoiding coke formation, high water contents may lead to catalyst and sorbent 
deactivation due to pore blocking [18]. On the contrary, lower WGFRs are not so problematic from the economic point of view since the glycerol produced through the biodiesel production process is composed by low amounts of water (3.2\% water, $40 \%$ fatty matter, $33 \%$ glycerol, $23 \%$ methanol and 3.8\% ash) [19]. Therefore, if lower WGFRs are employed, less water has to be added. Even if low WGFRs are not so advantageous in terms of hydrogen production, the use of a hybrid reaction-separation process like the one here described allows reducing the constraints imposed by such low water contents.

\subsubsection{Effect of Pressure}

Finally, the effect of pressure and hydrogen removal fraction on the yield of hydrogen and methane was analyzed, as can be seen in Fig. 7. It can be observed that when higher pressures are used, the yield of hydrogen suffers a negative effect, except for very high hydrogen removal fractions where constant hydrogen yields are observed. Such effect is once again related to the Le Chatelier's principle, because during GSR the total number of moles varies (eq. (1)). Therefore, when higher pressures are applied, the thermodynamic equilibrium will be shifted towards the production of a lower total number of moles. However, once the glycerol conversion was complete for all the range of pressures used, the decrease of the hydrogen yield cannot be associated to GSR. Instead, it is associated to the enhancement of the methanation reaction (eq. (4)), which consumes hydrogen and yields methane. As already stated, this is not valid for very high hydrogen removal fractions, where there is no methane. While at 1 atm, and considering $f_{\mathrm{CO}_{2}}=0.99$ and $f_{\mathrm{H}_{2}}=0.80$, the hydrogen and methane yields were 6.92 and 0.02 , respectively, at $5 \mathrm{~atm}$ the hydrogen yield decreased to 6.06 and the methane one increased to 0.23 .

In terms of carbon dioxide, its yield varies with pressure in a very similar way as hydrogen does (data not shown), for the reasons already mentioned. The production of carbon monoxide was once again negligible. Also, both RWGS (reverse of eq. (3)) and methanation (eq. (4)) control the process for all the range of pressures analysed, except when the formation of methane is completely inhibited. In this last case, WGS is the only relevant reaction. 
Regarding the effect of the hydrogen removal fraction, analogously to the previous cases, the hydrogen yield is favoured by increasing hydrogen removals, while methane suffers an opposite effect. The higher enhancement of the hydrogen yield is observed at the less thermodynamically favourable pressure (5 atm). It is, nonetheless, important to mention that the approach used here ignores the effect of pressure on the removal of both carbon dioxide and hydrogen, as well as in the reaction kinetics. It is known that pressure influences the driving force for carbon dioxide sorption and for hydrogen permeation through a hydrogen perm-selective membrane. Thus, higher pressures lead to higher carbon dioxide and hydrogen removal fractions, meaning that if high pressures are used in the SEMR, high hydrogen removals are attained and inherently higher hydrogen yields. Moreover, in the case of $f_{\mathrm{CO}_{2}}=0.99$ and $f_{\mathrm{H}_{2}}=0.80$, when the pressure increases from 1 to $5 \mathrm{~atm}$, the hydrogen yield at equilibrium decreases much less than for lower hydrogen removals, namely for $f_{\mathrm{H}_{2}}=0$ (SER). Consequently, the use of high pressures becomes advantageous after all due to the membrane effect.

\subsubsection{Coke Formation}

The formation of coke was target of investigation as well, since it is an undesired product that may affect the performance of the catalyst, sorbent and membrane during actual operation of the SEMR. It was observed that the formation of coke in the TR increases with the decrease of temperature (and so a higher WGFR would be required to completely avoid coke formation) during GSR, however at $600 \mathrm{~K}$ and $1 \mathrm{~atm}$ a WGFR of 4 is high enough to completely avoid coke formation. When carbon dioxide or hydrogen were removed from the reaction medium, opposite effects were observed. While the formation of coke decreases comparatively to the TR when carbon dioxide is removed from the reaction medium, when hydrogen is removed more coke is produced. For a carbon dioxide removal of $99 \%$, a WGFR of 1 is enough to completely avoid coke formation at temperatures between 600 and $800 \mathrm{~K}$ and $1 \mathrm{~atm}$. On the other hand, when $99 \%$ of hydrogen is removed, a WGFR of 4.5 is necessary to avoid carbon formation in the same temperature range and atmospheric pressure. Therefore, it allows us to conclude that most of coke is formed from methane through the reaction in eq. (8) (the low WGFRs allow the formation of 
methane, even at hydrogen and carbon dioxide removals of 99\%), being that the reactions in eqs. (7,9 and 10) are not compatible with the observed behavior and so they can be neglected.

It is therefore expected that when both products are removed from the reaction medium in the SEMR, an equilibrium between both opposite effects is reached. That was in fact observed, being that at $f_{\mathrm{CO}_{2}}=0.99$ and $f_{\mathrm{H}_{2}}=0.80$ a WGFR of 2.5 was enough to completely inhibit coke formation for all the range of temperatures and pressures tested. Even for a removal of $99 \%$ of both products, the stoichiometric WGFR of 3 was sufficient to avoid coke formation in all the temperature and pressure range. Therefore, it would be possible, from the thermodynamic point of view, to operate a SEMR for GSR under the temperature, WGFR and pressure ranges analysed in this study without having any problems with coke formation.

\section{Conclusions}

A thermodynamic analysis was carried out on the GSR for hydrogen production in a SEMR with in situ hydrogen and carbon dioxide simultaneous removal. At $800 \mathrm{~K}$, WGFR of 9, 1 atm and $f_{\mathrm{CO}_{2}}=0.99$ and $f_{\mathrm{H}_{2}}=0.80$, the hydrogen yield was 7 (maximum possible value). Regarding methane and carbon monoxide, no considerable production was observed under such conditions. Even though the SEMR presents higher hydrogen production than the SER and the MR and much higher hydrogen production than the TR, the difference is not very significative at higher temperatures compared to the two first configurations. However, this difference becomes much more meaningful with the decrease of temperature. At $600 \mathrm{~K}$, a hydrogen production of 5 moles/mole of converted glycerol was observed in the SEMR with $f_{\mathrm{CO}_{2}}=0.99$ and $f_{\mathrm{H}_{2}}=0.80$, while hydrogen productions of 1.83 and 2.62 moles/mole of converted glycerol were obtained in the SER and MR with the same removals, respectively. Therefore the advantages of the SEMR are more noticeable at lower temperatures and so, this new hybrid reactor configuration can be seen as a possible solution to lower the high operating temperatures necessary for the endothermic GSR. However, a proper judgement has to be done, because at lower temperatures longer reactors 
and longer and/or more permeable membranes would be necessary to compensate the decreased reaction kinetics and permeation rate.

In terms of WGFR and pressure effects, it is clear, from thermodynamic point of view, that higher WGFRs and lower pressures favor the production of hydrogen in the SEMR. Finnaly, it was observed that no coke was formed at WGFRs higher than 3.

\section{Acknowledgements}

Joel M. Silva acknowledges the Doctoral Program in Chemical and Biological Engineering from FEUP and the FCT for the PhD grant (PD/BD/52625/2014). M.A. Soria is grateful to the FCT for the postdoctoral grant (SFRH/BD/88444/2012). 


\section{References}

[1] H. Chen, T. Zhang, B. Dou, V. Dupont, P. Williams, M. Ghadiri, Y. Ding, International Journal of Hydrogen Energy, 34 (2009) 7208-7222.

[2] Y. Li, W. Wang, B. Chen, Y. Cao, International Journal of Hydrogen Energy, 35 (2010) 77687777.

[3] X. Wang, N. Wang, M. Li, S. Li, S. Wang, X. Ma, International Journal of Hydrogen Energy, 35 (2010) 10252-10256.

[4] C.V. Miguel, A. Mendes, S. Tosti, L.M. Madeira, International Journal of Hydrogen Energy, 37 (2012) 12680-12687.

[5] J.M. Silva, M.A. Soria, L.M. Madeira, in, Submitted.

[6] A.C.C. Chang, W.-H. Lin, K.-H. Lin, C.-H. Hsiao, H.-H. Chen, H.-F. Chang, International Journal of Hydrogen Energy, 37 (2012) 13110-13117.

[7] K.-H. Lin, W.-H. Lin, C.-H. Hsiao, H.-F. Chang, A.C.C. Chang, International Journal of Hydrogen Energy, 37 (2012) 13770-13776.

[8] A. Hanif, S. Dasgupta, S. Divekar, A. Arya, M.O. Garg, A. Nanoti, Chemical Engineering Journal, 236 (2014) 91-99.

[9] C.V. Miguel, R. Trujillano, V. Rives, M.A. Vicente, A.F.P. Ferreira, A.E. Rodrigues, A. Mendes, L.M. Madeira, Separation and Purification Technology, 127 (2014) 202-211.

[10] P.N. Sutar, P.D. Vaidya, A.E. Rodrigues, Chemical Engineering and Technology, 33 (2010) 1645-1649.

[11] C.K. Cheng, S.Y. Foo, A.A. Adesina, Industrial \& Engineering Chemistry Research, 49 (2010) 10804-10817.

[12] S. Adhikari, S. Fernando, S.R. Gwaltney, S.D. Filip To, R. Mark Bricka, P.H. Steele, A. Haryanto, International Journal of Hydrogen Energy, 32 (2007) 2875-2880.

[13] X. Wang, S. Li, H. Wang, B. Liu, X. Ma, Energy and Fuels, 22 (2008) 4285-4291.

[14] H. Chen, Y. Ding, N.T. Cong, B. Dou, V. Dupont, M. Ghadiri, P.T. Williams, Renewable Energy, 36 (2011) 779-788.

[15] S. Adhikari, S. Fernando, A. Haryanto, Energy and Fuels, 21 (2007) 2306-2310.

[16] N. Hajjaji, A. Chahbani, Z. Khila, M.-N. Pons, Energy, 64 (2014) 473-483.

[17] D. Mendes, S. Sá, S. Tosti, J.M. Sousa, L.M. Madeira, A. Mendes, Chemical Engineering Science, 66 (2011) 2356-2367.

[18] Martunus, Z. Helwani, A.D. Wiheeb, J. Kim, M.R. Othman, International Journal of Greenhouse Gas Control, 7 (2012) 127-136.

[19] M. Slinn, K. Kendall, C. Mallon, J. Andrews, Bioresource Technology, 99 (2008) 5851-5858. 


\section{Table Caption}

Table 1 - Reactions in equilibrium considered during the GSR thermodynamic simulations.

\begin{tabular}{|c|c|c|}
\hline Reaction & $\Delta \mathrm{H}_{\mathrm{r}}^{298 \mathrm{~K}}\left(\mathrm{~kJ} \cdot \mathrm{mol}^{-1}\right)$ & Reaction number \\
\hline $\begin{array}{l}\mathrm{C}_{3} \mathrm{H}_{8} \mathrm{O}_{3}+3 \mathrm{H}_{2} \mathrm{O} \rightleftharpoons 7 \mathrm{H}_{2}+3 \mathrm{CO}_{2} \\
\text { (GSR - glycerol steam reforming) }\end{array}$ & 128 & (1) \\
\hline $\begin{array}{l}\mathrm{C}_{3} \mathrm{H}_{8} \mathrm{O}_{3} \rightleftharpoons 3 \mathrm{CO}+4 \mathrm{H}_{2} \\
\text { (decomposition of glycerol) }\end{array}$ & 251 & $(2)$ \\
\hline $\begin{array}{l}\mathrm{CO}+\mathrm{H}_{2} \mathrm{O} \rightleftharpoons \mathrm{H}_{2}+\mathrm{CO}_{2} \\
\text { (WGS - water-gas shift) }\end{array}$ & -41 & (3) \\
\hline $\begin{array}{l}\mathrm{CO}+3 \mathrm{H}_{2} \rightleftharpoons \mathrm{CH}_{4}+\mathrm{H}_{2} \mathrm{O} \\
\text { (methanation) }\end{array}$ & -206 & (4) \\
\hline $\mathrm{CO}_{2}+4 \mathrm{H}_{2} \rightleftharpoons \mathrm{CH}_{4}+2 \mathrm{H}_{2} \mathrm{O}^{\mathrm{a}}$ & -165 & (5) \\
\hline $\begin{array}{l}2 \mathrm{CO}+2 \mathrm{H}_{2} \rightleftharpoons \mathrm{CH}_{4}+\mathrm{CO}_{2}{ }^{\mathrm{b}} \\
\text { (dry reforming of methane) }\end{array}$ & -247 & (6) \\
\hline $\begin{array}{l}2 \mathrm{CO} \rightleftharpoons \mathrm{CO}_{2}+\mathrm{C} \\
\text { (Boudouard reaction) }\end{array}$ & -172 & (7) \\
\hline $\begin{array}{l}\mathrm{CH}_{4} \rightleftharpoons 2 \mathrm{H}_{2}+\mathrm{C} \\
\text { (methane cracking) }\end{array}$ & 75 & $(8)$ \\
\hline $\begin{array}{l}\mathrm{CO}+\mathrm{H}_{2} \rightleftharpoons \mathrm{H}_{2} \mathrm{O}+\mathrm{C} \\
\text { (carbon monoxide reduction) }\end{array}$ & -131 & (9) \\
\hline $\begin{array}{l}\mathrm{CO}_{2}+2 \mathrm{H}_{2} \rightleftharpoons 2 \mathrm{H}_{2} \mathrm{O}+\mathrm{C} \\
\text { (carbon dioxide reduction) }\end{array}$ & -90 & $(10)$ \\
\hline
\end{tabular}




\section{Figures Captions}
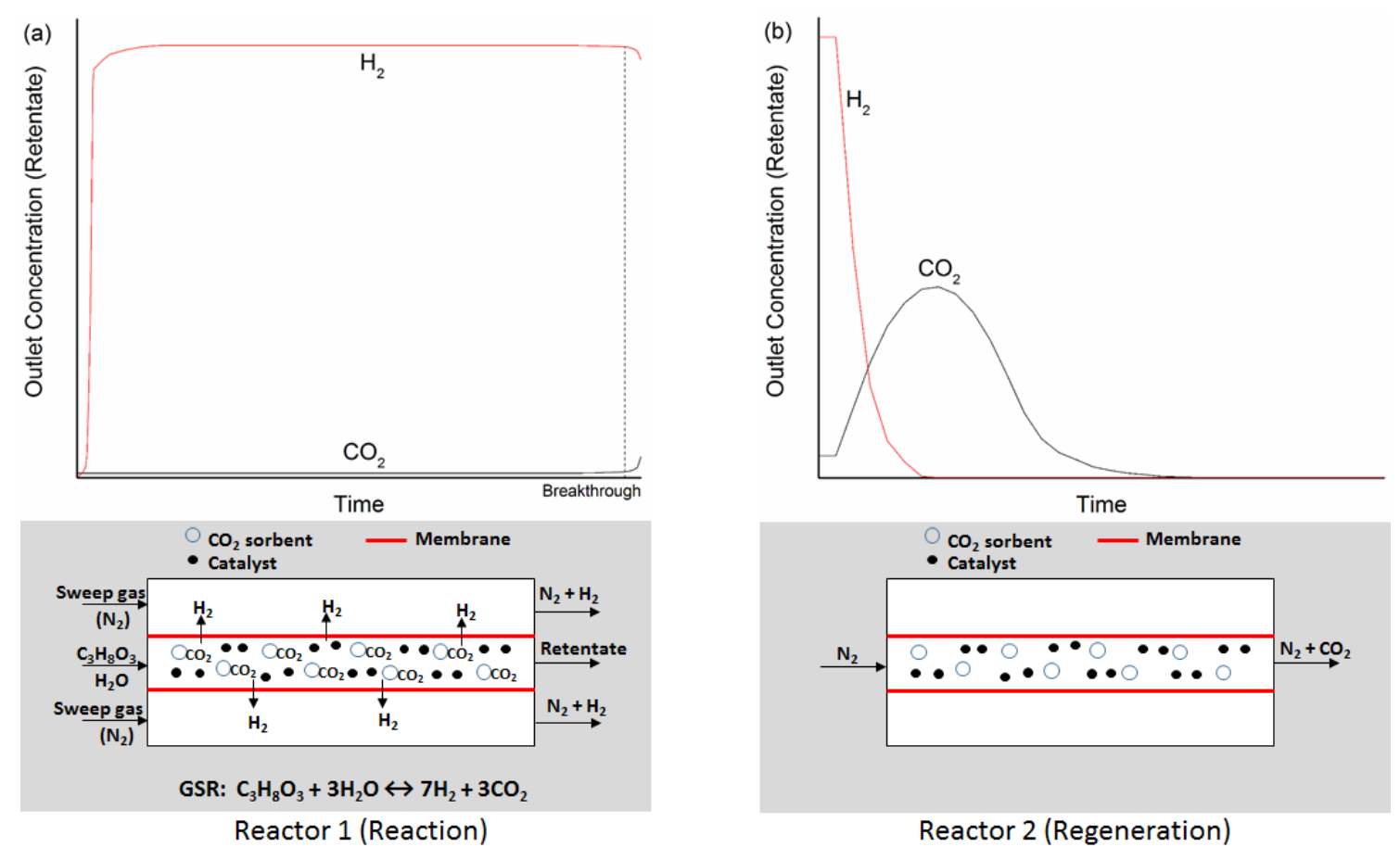

Fig. 1 - Schematic view of the conceived SEMR based on 2 parallel reactors configuration for continuous operation and corresponding outlet concentrations histories in the retentate stream during (a) reaction and (b) regeneration stages. 


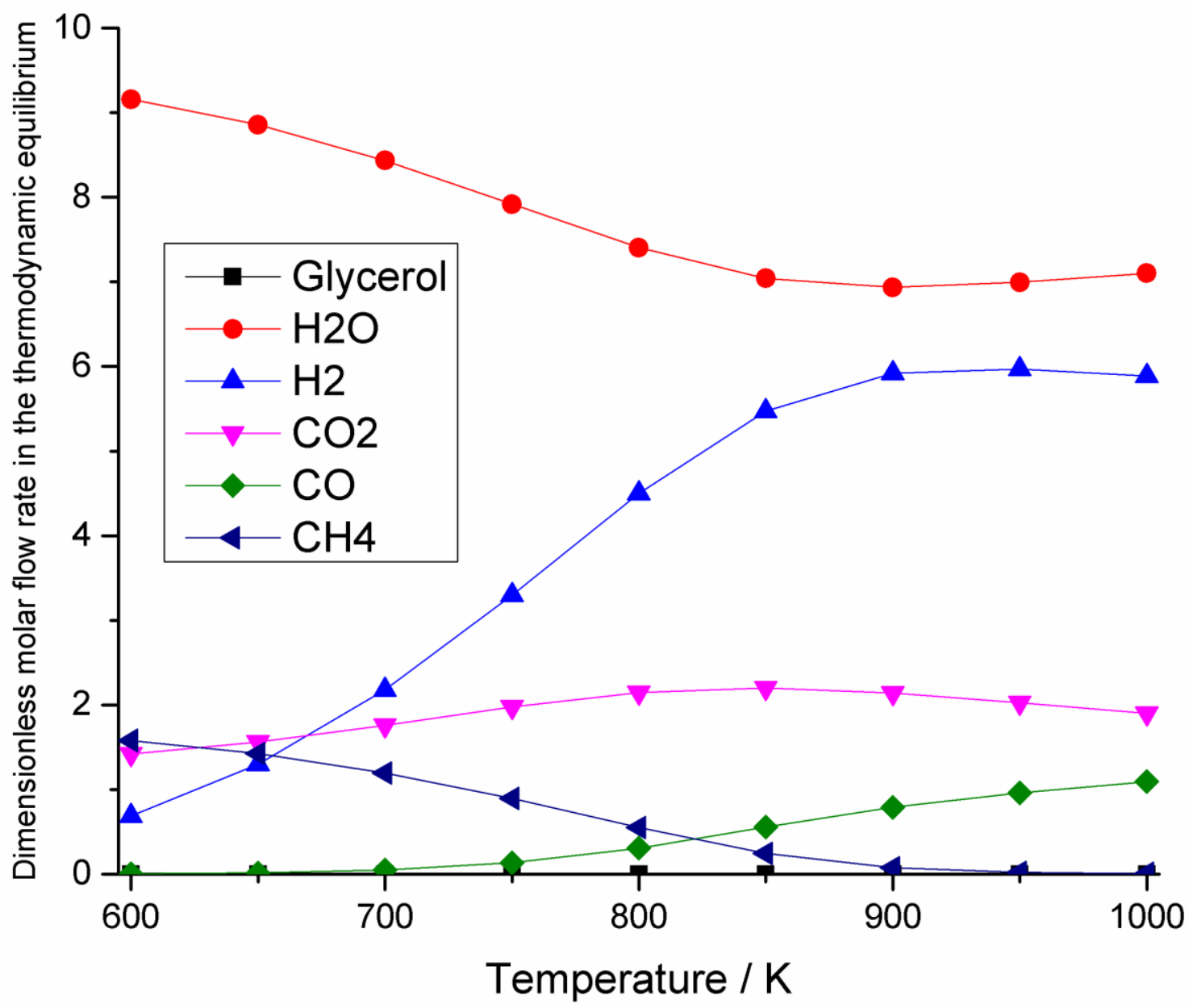

Fig. 2 - Effect of temperature on the thermodynamic equilibrium composition of a traditional reactor at WGFR of 9 and 1 atm. 

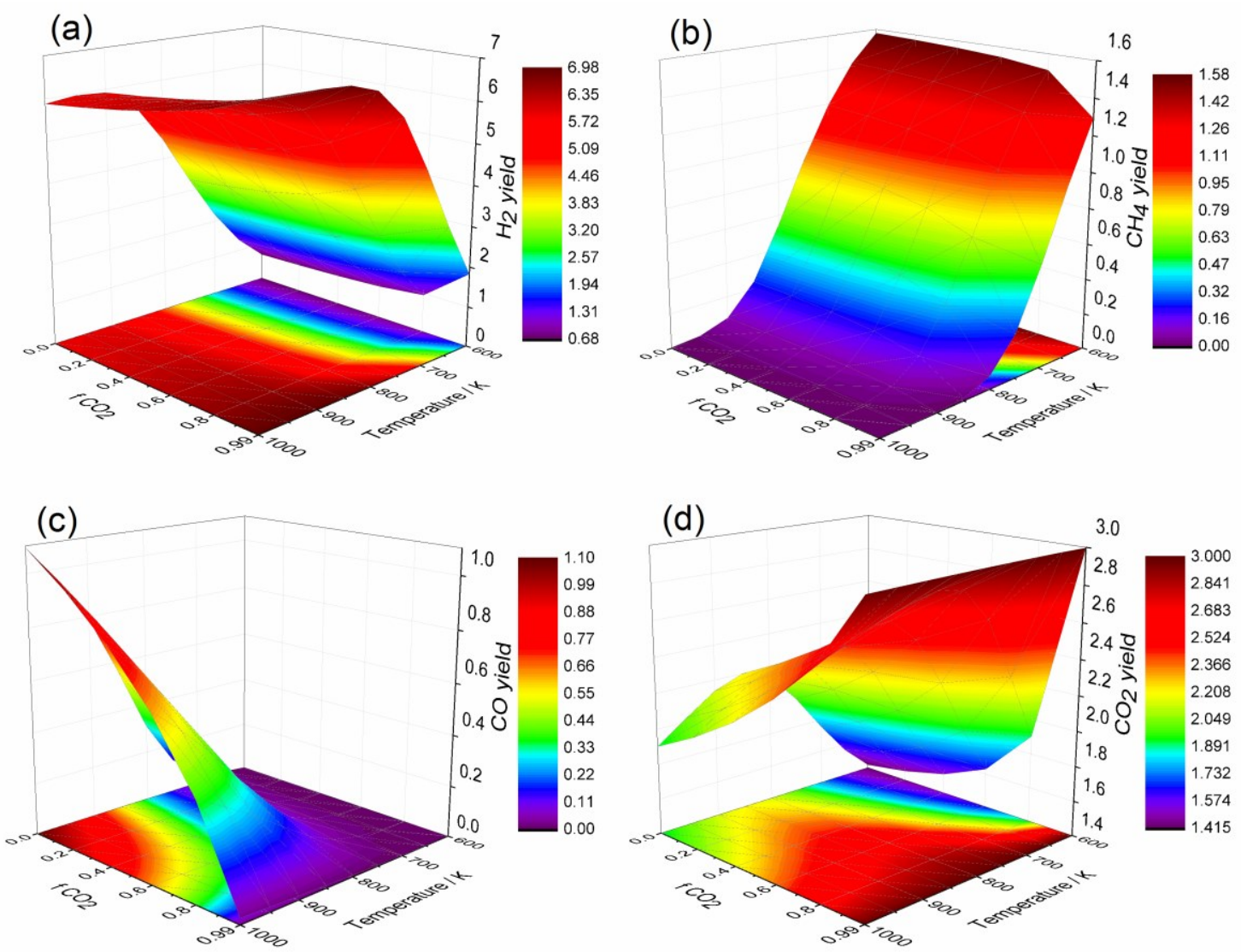

Fig. 3 - Effect of temperature and carbon dioxide removal fraction of the SER on the yield of (a) hydrogen, (b) methane, (c) carbon monoxide and (d) carbon dioxide, at WGFR of 9 and 1 atm. 
(a)

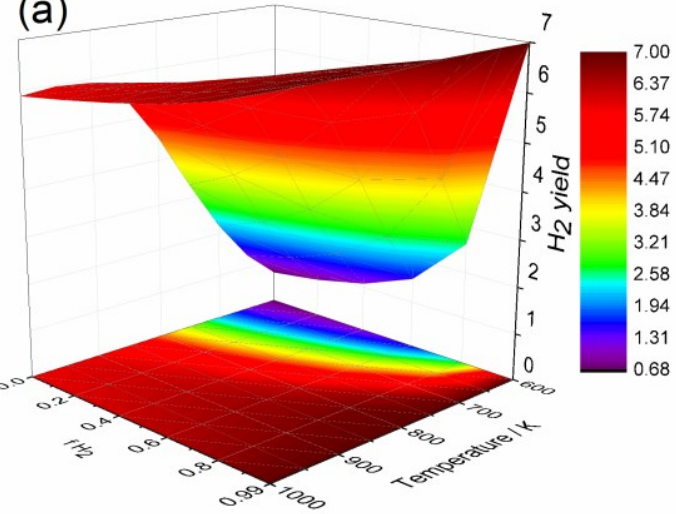

(c)

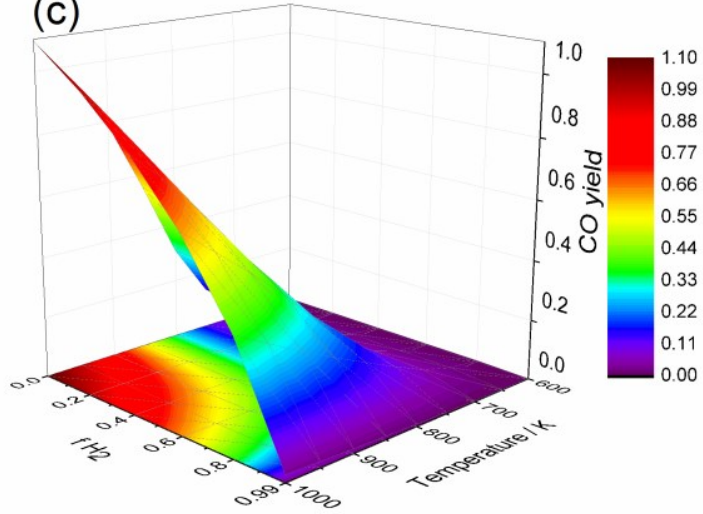

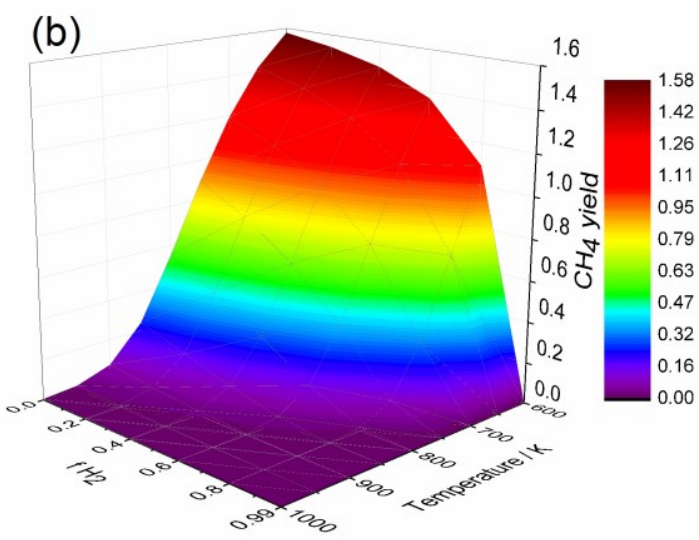

(d)

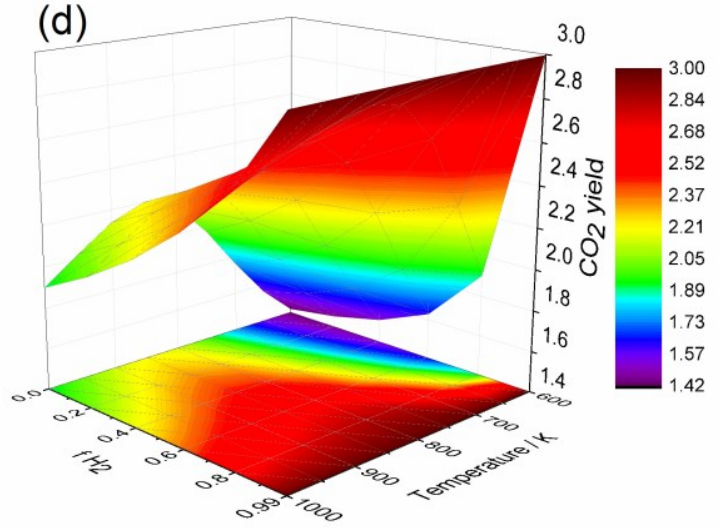

Fig. 4 - Effect of temperature and hydrogen removal fraction of the MR on the yield of (a) hydrogen, (b) methane, (c) carbon monoxide and (d) carbon dioxide, at WGFR of 9 and 1 atm.
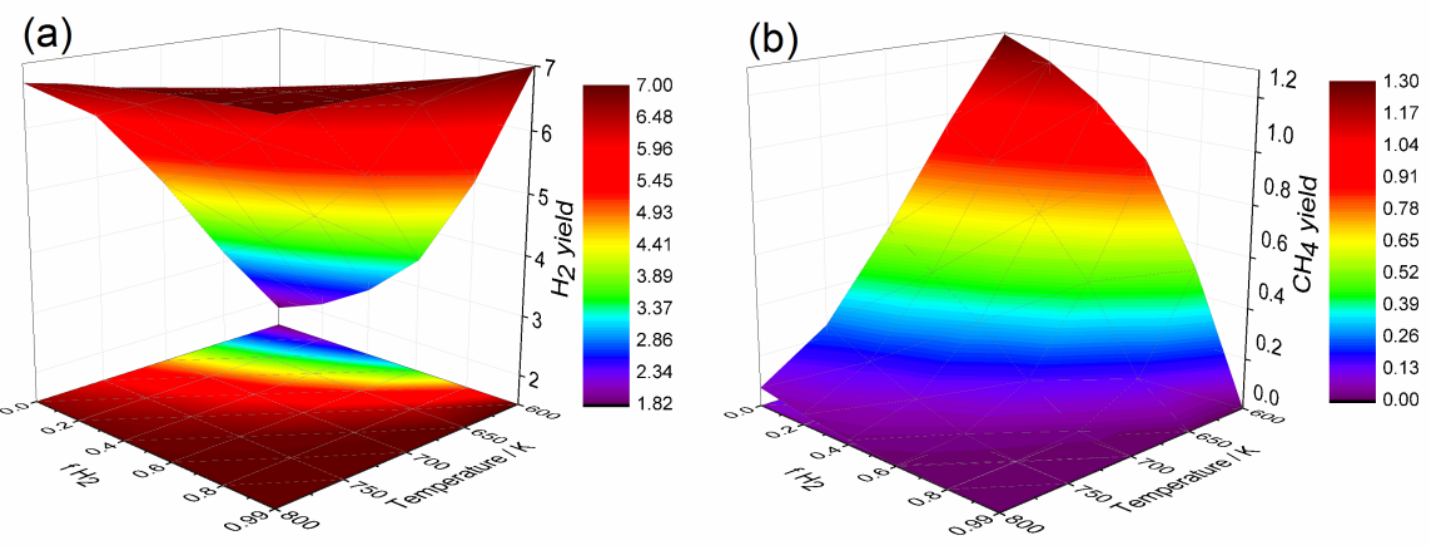

Fig. 5 - Effect of temperature and hydrogen removal fraction on the yield of (a) hydrogen and (b) methane at WGFR of 9, 1 atm and carbon dioxide removal fraction of 0.99 in a SEMR. 

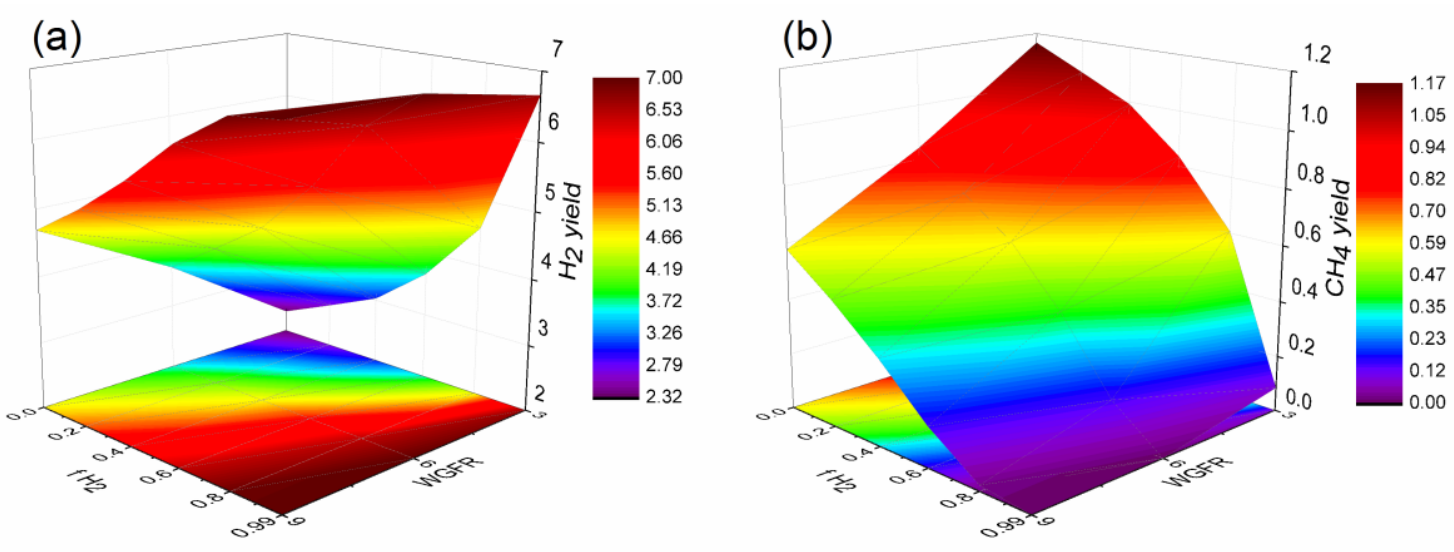

Fig. 6 - Effect of WGFR and hydrogen removal fraction on the yield of (a) hydrogen and (b) methane at $700 \mathrm{~K}, 1 \mathrm{~atm}$ and carbon dioxide removal fraction of 0.99 in a SEMR.
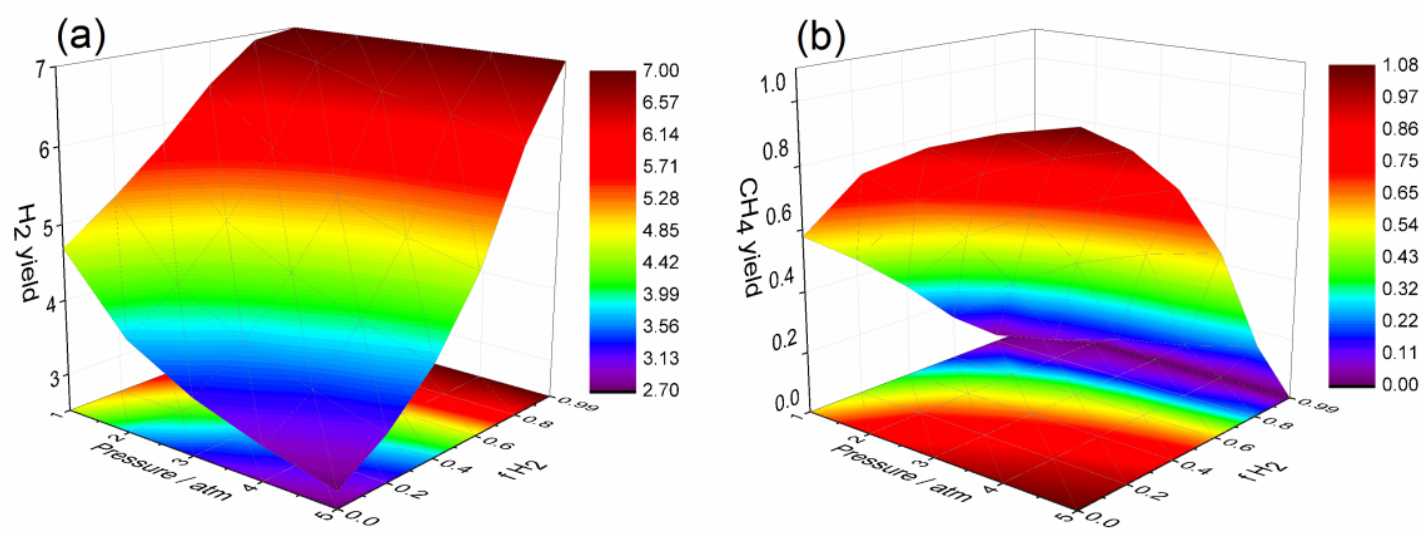

Fig. 7 - Effect of pressure and hydrogen removal fraction on the yield of (a) hydrogen and (b) methane at $700 \mathrm{~K}$, WGFR of 9 and carbon dioxide removal fraction of 0.99 in a SEMR. 